CARNETS OE Carnets de géographes

GÉOGRAPHES.

5 | 2013

Géographie humanimale

\title{
Les conflits hommes/animaux sauvages sous le regard de la géographie
}

Cadre territorial, perceptions et dimension spatiale

\section{Guillaume Marchand}

\section{OpenEdition}

\section{Journals}

Édition électronique

URL : http://journals.openedition.org/cdg/1070

DOI : $10.4000 / \mathrm{cdg} .1070$

ISSN : 2107-7266

\section{Éditeur}

UMR 245 - CESSMA

\section{Référence électronique}

Guillaume Marchand, «Les conflits hommes/animaux sauvages sous le regard de la géographie », Carnets de géographes [En ligne], 5 | 2013, mis en ligne le 01 janvier 2013, consulté le 19 avril 2019. URL : http://journals.openedition.org/cdg/1070 ; DOI : 10.4000/cdg.1070

\section{(c)}

La revue Carnets de géographes est mise à disposition selon les termes de la Licence Creative Commons Attribution - Pas d'Utilisation Commerciale - Pas de Modification 4.0 International. 


\title{
LES CONFLITS HOMMES/ANIMAUX SAUVAGES SOUS LE REGARD DE LA GEOGRAPHIE \\ Cadre territorial, perceptions et dimension spatiale
}

\author{
GUILLAUME MARCHAND \\ Géographe \\ Professeur visitant étranger à l'Université Fédérale de l'Amazonas, \\ Boursier de la Coordenação de Aperfeiçoamento de Pessoal de Nível Superior (CAPES) \\ gaelmarch@yahoo.fr
}

\begin{abstract}
Résumé
Les conflits entre les hommes et la faune sauvage se révèlent par un ensemble de nuisances (destruction de cultures, attaques d'animaux d'élevage, compétitions pour les ressources naturelles, atteintes à la vie humaine) provoquées par différentes espèces animales. En réponse à ces nuisances ou pour tenter de les prévenir, les espèces responsables sont le plus souvent blessées ou tuées par les populations locales, même si d'autres solutions sont explorées avec l'aide des pouvoirs publics et des ONG (délocalisations, compensations, investissements dans la prévention). Dans plusieurs rapports publiés au cours des années 2000, la FAO a souligné une augmentation de ces conflits à l'échelle mondiale, faisant état de la diversité des cas de figure rencontrés d'une région du globe à l'autre. Actuellement, les sociétés confrontées à ces problèmes se posent de nombreuses questions sur leurs causes, leurs impacts sociaux et environnementaux mais aussi sur les solutions à mettre en œuvre pour obtenir une cohabitation plus pacifique. L'objectif de cet article est de montrer que ces interrogations peuvent être intégrées au champ de la géographie et de présenter les réponses que cette discipline peut apporter. Nous verrons alors que cette science a des atouts à faire valoir, que ce soit par ses méthodes d'analyse développées autour des conflits environnementaux, ses travaux sur la représentation des animaux ou son savoir-faire dans l'étude des facteurs spatiaux d'une grande variété de phénomènes.
\end{abstract}

\footnotetext{
Abstract

Conflicts between humans and wildlife refer to a range of nuisances (destruction of crops, livestock attacks, competition for natural resources, damage to human life) caused by different species. In response to these disturbances or in on order to prevent them,
} 
responsible species are often injured or killed by local populations, even if other solutions are explored with the help of government and NGOs (translocations, compensations, investments in prevention). In several reports published in the 2000s, the FAO noted an increase in conflicts around the world and the diversity of local situations. Human societies facing these issues work on causes, social and environmental impacts and solutions to reach a more peaceful coexistence. This paper shows how geography can be used to deal with human-wildlife conflicts through environmental conflict analysis, work on animal representation or study of spatial factors.

\section{Introduction}

Au cours des années 1990, la biologie de la conservation s'est intéressée sérieusement aux problèmes inhérents à la coexistence de groupes humains et d'espèces animales sauvages sur un même espace, les regroupant sous le terme de human/wildlife conflicts (Woodroffe et al., 2005). L'idée était que les préjudices subis par les populations humaines (destructions de cultures, attaque de bétail, risques de blessure ou de décès...) pouvaient avoir un impact non négligeable sur la protection des espèces problématiques, notamment à cause des risques d'abattage qui pesaient sur des spécimens menacés à divers degrés d'extinction. Dès lors, les études autour des conflits hommes/animaux sauvages n'ont eu de cesse de se multiplier, mobilisant un grand nombre de disciplines en raison de la diversité des facteurs sociétaux et environnementaux entrant en ligne de compte ${ }^{1}$. Parmi ces différentes disciplines, figure, certes encore de façon modeste, la géographie. L'objectif de cet article est de montrer pourquoi cette discipline peut intégrer la question des conflits hommes/faune sauvage dans son champ d'étude et quels peuvent être ses apports dans ce domaine. Notre propos sera organisé en deux parties. La première reviendra sur les éléments permettant de justifier l'inscription des conflits hommes/faune sauvage dans le champ de la géographie. La seconde abordera dans le détail les types d'analyses que cette science peut fournir autour de trois questions identifiées comme fondamentales dans la littérature scientifique consacrée au sujet : I'analyse du cadre territorial dans lequel s'inscrivent les conflits, la façon dont ils sont perçus par les populations locales et leur dimension spatiale (conditions géographiques favorables aux interactions négatives entre espèces ou à leurs résolutions). Le choix de ce triptyque sera justifié au cours du développement de notre propos.

\section{Les conflits hommes/animaux sauvages: une nécessaire appréhension conjointe des dimensions humaines et animales}

Les conflits hommes/animaux sauvages suscitent un intérêt croissant dans nos sociétés. Au niveau international, la FAO constatant une augmentation de ces conflits à travers le monde pose un certain nombre de questions sur leurs causes/conséquences et les moyens à mettre en œuvre pour une cohabitation plus pacifique (Distefano, 2005 ; Lamarque et al., 2010).

\footnotetext{
${ }^{1}$ Pour avoir une idée des ressources bibliographiques disponibles dans ce domaine consulter la page web : http://www.peopleandwildlife.org.uk/biblio.shtml
} 
Autant de questionnements auxquels les différentes disciplines scientifiques sont invitées à répondre.

\section{Les conflits hommes/faune sauvage : définition d'un conflit environnemental presque comme les autres}

Avant toute chose, même si nous avons esquissé en introduction une définition des conflits hommes/faune sauvage, il semble utile d'expliciter davantage ce terme pour la suite de notre propos. Selon Madden (2004 : 2) : " II y a conflit quand les besoins et le comportement de la faune sauvage ont un impact négatif sur les objectifs des humains ou quand les objectifs des humains ont un impact négatif sur la faune » (notre traduction).

Du côté des impacts négatifs de la faune sur les sociétés humaines, on recense généralement les attaques de cultures agricoles ou forestières par des herbivores/frugivores mais aussi des attaques de carnivores sur les animaux d'élevage ou sur des personnes. Ces interactions négatives sont alors considérées comme directes dans le sens où ces différentes espèces détruisent du capital humain (surfaces agricoles, habitations) et/ou portent atteinte à l'intégrité physique des hommes et des animaux sous leur protection. II existe également des interactions négatives indirectes ou plus insidieuses. C'est le cas lorsque la faune sauvage se voit reprochée (à tort ou à raison) de surconsommer les ressources naturelles normalement utilisées par des groupes humains (poissons, gibier, fruits et semences) ou de perturber leur prélèvement. Enfin, il est important de garder à l'esprit que la seule présence d'animaux dangereux ou difficilement contrôlables à proximité d'installations humaines provoque des réactions plus ou moins vives de contestation en raison de la gêne et des risques qu'ils occasionnent. Du côté des impacts négatifs sur la faune, les mesures portant atteinte à l'intégrité physique de l'animal sont les plus souvent citées. Les solutions les plus prisées par les populations locales pour empêcher, réduire ou résoudre les conflits étant d'empoisonner, de blesser ou d'abattre soit de manière sélective (poursuite de l'individu fautif) soit de façon aléatoire (un quelconque représentant de l'espèce problématique). La dégradation des habitats et le prélèvement de ressources naturelles par les hommes au détriment des autres espèces entrent également dans la définition donnée précédemment.

Pour Johansson (2009), les conflits naissent généralement de deux types de transgression. Dans un premier temps, lorsque l'animal sort de la place réelle (une aire protégée, une réserve, un enclos) ou symbolique (une espèce $X$ doit "normalement " se trouver dans tel ou tel type d'espace) que la société lui a assigné. Dans un second temps, lorsque l'homme envahit ou s'aventure dans les espaces occupés par les animaux sauvages, surtout que certaines espèces les défendent farouchement contre divers types d'intrus ( $d$ 'où l'utilisation du terme "territoire ${ }^{2}{ }^{2}$. Au vu de ce que nous avons écrit auparavant, il semble opportun de rajouter que les conflits peuvent également émerger en dehors de ces transgressions. Les animaux peuvent être pourchassés, abattus, déplacés, contrôlés ou faire l'objet de

\footnotetext{
${ }^{2}$ Pour les sciences naturelles, le territoire d'un animal correspond à une portion d'espace délimitée par différents marqueurs biologiques (urine, fèces, sécrétions glandulaires...) protégée contre des individus de la même espèce (sauf ceux du sexe opposé lors de saisons de reproduction) mais aussi souvent contre d'autres espèces (défense des petits, compétitions pour l'usage de certaines ressources). Ce territoire regroupe différents espaces (zones d'alimentation, de repos, de reproduction) qui peuvent être distincts (configuration en archipels) ou confondus (superposition des différentes fonctions sur un même espace). Voir Milhaud (2005).
} 
réclamations sans avoir véritablement commis de forfait, mais en raison des menaces potentielles qu'ils représentent. La situation de conflit peut donc être la conséquence de dommages matériels et de compétitions inter-espèces avérés, mais la façon dont les animaux sont perçus et le ressenti jouent également un rôle de premier plan.

Les conflits hommes/faune sauvage peuvent être intégrés à la famille des conflits environnementaux (Torre, 2010) dans le sens où un facteur environnemental, ici la présence d'espèces pouvant potentiellement porter préjudice aux sociétés humaines, provoque des réactions plus ou moins violentes en leur sein (revendications et demandes d'intervention auprès des pouvoirs publics, débats entre acteurs, mesure de représailles envers les espèces incriminées). Dans bien des cas, les conflits hommes/animaux sauvages sont considérés comme de simples "conflits entre hommes au sujet des animaux ". On insiste alors sur le faits que les affrontements entre différents groupes d'acteurs (personnalités politiques, associations, citoyens lambda, ONGs...) sont liés à de vives divergences quant à la manière de penser la protection de l'environnement, de vivre ou d'utiliser un espace donné mais aussi de percevoir les espèces à problèmes (Luxereau, 2004 ; Collomb, 2009). Toutefois, ils ne sauraient se limiter à cette vision qui relègue au second plan le versant animal du problème. L'une des principales originalités des conflits hommes/animaux sauvages est que l'animal est un protagoniste à part entière avec des stratégies qui lui sont propres dans sa manière d'occuper l'espace et de se jouer, avec une fréquence et une intensité variables, d'une part, des frontières imposées par les groupes humains et, d'autre part, des représentations à son égard (Philo et Wilbret, 2000 ; Emel et Urbanik, 2010).

\section{Enjeux sociaux et environnementaux des conflits hommes/sauvages}

Nous avons insisté dans la définition donnée dans la partie précédente sur la dimension humaine et animale des conflits environnementaux, nous présenterons ici de façon concise quelques-uns des problèmes sociaux et environnementaux qui leur sont inhérents.

Du point de vue social, lesdits conflits ont de nombreuses incidences sur les conditions de développement humain. Une fois cumulées, les destructions de surfaces cultivées et les attaques de bétail par la faune sauvage se chiffrent souvent en millions d'euros à l'échelle d'une nation (Lamarque et al., op.cit). Si ces conflits concernent tant les grands producteurs fortement capitalisés que les petits paysans, les incidents ont souvent un impact plus important dans le second groupe. Cela est dû au manque de moyens pour se prémunir des attaques mais aussi à la vulnérabilité économique ambiante : production sujette à des aléas divers, capacité de réaction aux crises limitée (absence de liquidités, impossibilité d'investissement). Dans un grand nombre de pays, surtout ceux en développement, le problème est accentué par l'absence de mesures compensatoires (indemnisations) ou d'aides à la prévention (système d'assurance, clôture des parcs et réserves). Que ce soit pour les zones rurales de I'hémisphère Nord ou celles de I'hémisphère Sud, ces espaces sont soumis à une multitude de problèmes: difficultés à vendre la production, concurrence d'autres formes d'occupation de l'espace, difficultés d'accès à certains services fondamentaux etc. Par conséquent, les sommes investies pour protéger les espèces problématiques ou pour réparer leurs dégâts sont mal vécues ou considérées comme injustes car elles pourraient être utilisées pour résoudre les problèmes des populations locales (Benhammou et Dangléant, 2009). Enfin, il est important de garder à l'esprit que certaines espèces portent atteinte à la vie humaine. Selon Thirgood et al. (2005), les décès 
liés aux méga-herbivores (éléphants et hippopotames ${ }^{3}$ ) sont à peu près équivalents à ceux liés aux carnivores. À titre d'exemple, au Kenya, on dénombre 221 victimes de l'éléphant entre 1990 et 1997 contre 250 pour les félins (Panthera leo et Panthera pardus) et les crocodiles (différentes espèces du genre Crocodylus). D'une manière générale, les décès liés aux animaux sont plus nombreux dans les pays en développement que dans les pays développés, outre une plus forte proportion d'espèces potentiellement dangereuses, les densités de population rurale, la présence de vastes aires protégées et les formes d'agropastoralisme pratiquées peuvent expliquer ces différences.

Du point de vue environnemental, certaines des espèces classées comme nuisibles et dûment combattues par les populations locales sont également menacées d'extinction, comme l'once (Uncia uncia) ou les différentes branches asiatiques de Panthera tigris (Inskip et Zimmermann, 2009). Ces espèces sont déjà fragilisées par la disparition de leur habitat, par leur utilisation par la médecine traditionnelle chinoise ou encore par la raréfaction de leurs proies habituelles. Ainsi, l'utilisation généralisée du contrôle létal pour résoudre les problèmes qu'elles provoquent (attaque de bétail pour l'once, attaque humain/bétail pour le tigre) accroît la menace qui pèse sur elles. Il est utile de rappeler que même si elles s'avèrent gênantes, les espèces problématiques offrent "gratuitement " de nombreux services (agro)écosystémiques pour les territoires qu'elles occupent. Les carnivores évoqués cidessus régulent les rongeurs et herbivores qui s'attaquent aux cultures. Par conséquent, leur disparition peut avoir des conséquences directes sur les productions agricoles puisque les nuisibles devront être éliminés par des moyens plus coûteux (pesticides, poisons).

Le dernier enjeu (et de taille) a trait à la cohabitation hommes/espèces sauvages en minimisant les impacts évoqués jusqu'à présent. Une bonne partie de la littérature technicoscientifique est consacrée à la recherche de solutions alternatives au contrôle létal qu'elles soient préventives (clôtures, répulsifs, cloisonnement des troupeaux, système d'assurance contre les attaques), éducatives (sensibilisation aux enjeux de protection) ou compensatoires (indemnisations des préjudices subis, projets de développement socioéconomiques ${ }^{4}$...). Ces solutions sont éminemment liées aux conditions locales dans le sens où ce qui fonctionne dans un endroit donné et pour une espèce donnée ne vaut pas forcément ailleurs et pour une autre espèce. Par exemple, les clôtures balisées de drapeaux (fladry barriers) ont été mobilisées pour éviter les attaques des loups nord-américains (Canis lupus) sur les animaux d'élevage, partant du principe que les représentants européens de l'espèce étaient intimidés par les bouts de tissus en mouvement. Toutefois, les résultats obtenus par Shivik et al. (2003) sur le territoire américain ont été mitigés. Des loups faméliques ou téméraires n'hésitaient pas à franchir cet obstacle voyant que celui-ci ne présentait pas de réel danger pour leur propre existence. Ils furent rapidement imités par leurs semblables, ce qui a ralenti les prédations sur le bétail seulement pendant quelques mois. De même, les solutions proposées n'ont pas la même acceptation en fonction des populations humaines rencontrées, celle-ci dépendant de nombreux critères tels que les

\footnotetext{
${ }^{3}$ Pour l'éléphant, il s'agit des espèces du genre Loxodonta, pour l'hippopotame Hippopotamus amphibius

${ }^{4}$ L'ONG Snow Leopard Trust dédiée à la protection de la panthère des neiges vend sur sa page internet des produits fabriqués par des communautés cohabitant avec ce grand félin. Il s'agit principalement d'artisanat réalisé à partir de laines ou de peaux d'animaux domestiques. Pour que les produits soient vendus par l'ONG, les populations doivent s'engager à ne pas abattre de panthères sous peine de voir le partenariat être rompu : http://www.snowleopard.org/programs/communitybasedconservation/sle
} 
revenus (les méthodes utilisant des répulsifs sonores, visuels ou tactiles sont très coûteuses) ou encore la sensibilité individuelle au monde animal.

Le zonage fait partie de ces solutions souvent mises en avant mais controversées (Larson, 2008). Ces plans d'aménagement consistent à implanter une ou des "zones cœur " où les espèces animales sont strictement protégées et, à leurs périphéries, des zones où les espèces problématiques peuvent potentiellement être abattues (en fonction du statut de l'espèce et des autorisations particulières fournies par les autorités). Ce type de proposition suscite des réactions diverses (Mauz, 2002a; Bobbé, 2006). D'un côté, les partisans des espèces problématiques perçoivent négativement l'autorisation du contrôle létal et craignent que les zonages ne prennent pas en compte les besoins spatiaux des animaux. Rappelons que certaines espèces nécessitent une aire vitale de plusieurs milliers de $\mathrm{km}^{2}$, ce qui est souvent impossible à mettre en œuvre en raison des besoins humains (terres de parcours, expansion urbaine, fronts pionniers agricoles). D'un autre côté, les détracteurs des espèces problématiques ne sont pas toujours satisfaits des autorisations d'abattage (qu'ils souhaiteraient plus permissives) et du fait de voir leurs propres usages de l'espace contraints, en partie, selon des critères biologiques et écologiques. Enfin, les zonages soulèvent de nombreuses questions quant à l'efficacité réelle d'un partage de l'espace. L'idée que les animaux transgressent les frontières qui leur sont imposées est unanime (Emel et Urbanik, op.cit), il en va de même avec les populations humaines. Si l'on prend l'exemple des invasions de terres protégées, celles-ci sont monnaies courantes dans le monde entier et pour des motifs divers. En Afrique, les aires protégées constituent des refuges en cas de sécheresse (pacage des bêtes) ou de troubles politiques (Binot et al., 2006). Au Brésil, cellesci sont occupées par les mouvements de réforme agraire radicaux et des latifundiaires, considérant qu'elles nuisent au développement socio-économique du pays (GTA, 2008). Par conséquent, de nombreuses questions demeurent en suspens quant à l'efficacité de cette partition de l'espace afin de réduire les conflits hommes/faune sauvage. Questions auxquelles la géographie peut apporter des réponses à travers ces différents outils cartographiques et d'analyse spatiale.

Au vu de ce qui a été évoqué précédemment, la géographie possède de nombreux atouts à faire valoir dans l'étude des conflits hommes/animaux sauvages. Premièrement, parce que ces derniers renvoient à des questions intégrées à son champ disciplinaire, telles que les interactions hommes/environnement ainsi que leurs variations dans le temps et l'espace. Deuxièmement, parce que les sciences naturelles qui ont tenu pendant longtemps le monopole des études sur ces conflits multiplient les appels au dialogue interdisciplinaire afin d'améliorer les connaissances sur leurs dimensions sociales, culturelles, politiques, économiques et spatiales. La géographie peut aisément répondre à cet appel à la collaboration, étant par essence une "science d'interface " habituée à l'étude systémique de facteurs de différentes natures ainsi qu'aux échanges avec les autres disciplines (Bertrand, 2002). La partie suivante de notre propos est destinée à montrer comment cette question a été traitée jusqu'à présent dans les travaux géographiques et quels sont les éléments à approfondir ou à explorer. 


\section{La géographie au service de l'étude des conflits hommes/animaux sauvages}

Depuis la publication du livre de Philo et Wilbret (2000), les études géographiques traitant de façon directe ou indirecte de la question animale se sont multipliées, particulièrement en Angleterre, en Amérique du Nord et en Australie (Emel et Urbanik, op.cit.). En France, malgré une réticence ancienne des géographes à parler des animaux (Milhaud, 2005), un dossier spécial de la revue Espaces et Sociétés publié en 2002 (sous la direction de Staszak) a réaffirmé la légitimité de cet objet d'étude dans le champ disciplinaire et a ouvert la voie à d'autres initiatives. Dans cet univers, on retrouve un certain nombre de travaux traitant directement ou indirectement ${ }^{5}$ des conflits hommes/animaux sauvages. L'objectif de cette partie est de montrer comment ces recherches et celles portant sur d'autres domaines peuvent contribuer à améliorer les connaissances autour de cet objet d'étude. Au vu de la littérature parcourue, nous avons identifié trois champs de compétence qui peuvent s'avérer utiles, le premier fait référence à l'étude des conditions territoriales des conflits, le second à l'analyse des perceptions et des représentations liées à l'animal et le troisième à la mise en évidence du volet spatial des conflits et à leur cartographie.

\section{La géographie des conflits : replacer les problèmes liés à l’animal dans une dynamique territoriale}

Des auteurs comme Madden (op. cit.) ont souligné la nécessité de replacer les conflits hommes/animaux sauvages dans une perspective territoriale en s'intéressant aux particularités locales ou aux traits communs pouvant être rencontrés d'une région à l'autre. Nous avons vu dans la partie précédente que les configurations territoriales influencent grandement la nature des conflits, leur gravité et les méthodes à mettre en place pour les résoudre. Par "configuration territoriale" nous faisons référence à la combinaison de facteurs aussi divers que: l'état de l'environnement (disponibilité des ressources pour humains et animaux), le type d'espèce rencontrée (les herbivores ne provoquant pas les mêmes problèmes que les carnivores), les activités humaines pratiquées (les conflits sont en général plus vivaces lorsque le secteur primaire est important), la façon dont sont perçus les animaux ainsi que les formes d'intervention pour faciliter la cohabitation. Cette mise en perspective des conflits selon les configurations territoriales est une pratique régulière de la géographie dédiée à l'étude des conflits environnementaux (Guyot 2003; Laslaz 2005; Goeury 2010 ; Torre, 2010). En faisant une synthèse des réflexions développées dans ces différents articles, il est possible de définir les contours d'une grille de lecture quant aux conditions territoriales des conflits avec la faune sauvage qui serait articulée en quatre points.

Le premier point invite à s'intéresser aux dynamiques territoriales qui sont à l'origine des conflits ou qui ont été révélées par ces derniers. Par exemple, les créations d'aires protégées en divers endroits du globe se sont accompagnées d'un accroissement démographique des

\footnotetext{
${ }^{5}$ Par indirectement, nous faisons référence aux travaux qui n'emploient pas telle quelle l'expression « conflits homme/faune sauvage " mais qui abordent des problèmes qui entrent dans cette catégorie (oppositions quant au partage de l'espace, perturbations des conditions de vie par les animaux, affrontements d'acteurs autour de certaines espèces jugées problématiques...).
} 
populations animales et conséquemment d'une augmentation des risques d'interactions négatives avec les groupes humains vivant en leur sein ou à proximité (Sogbohossou et al., 2011). En France, les opinions négatives à l'encontre du loup dans les régions montagnardes sont exacerbées par les difficultés rencontrées localement (crise de l'élevage ovin, problèmes d'accès à certains services).

Le deuxième point traite de l'analyse des jeux d'acteurs, ce qui revient à étudier les jeux d'alliances et d'oppositions mais aussi les discours et les stratégies qui les sous-tendent. En France, Mounet (2007) a mis en évidence que les agriculteurs et les chasseurs se rencontraient dans leur opposition au loup (respectivement inquiets pour les brebis et les populations d'ongulés sauvages) mais s'opposaient autour du sanglier ${ }^{6}$ (les destructions de cultures provoquées par ce dernier étant imputées à l'incapacité des chasseurs à gérer l'espèce). En faisant référence aux jeux d'acteurs, il est nécessaire de considérer l'animal comme un protagoniste à part entière qui possède des logiques qui lui sont propres et dont les actions résultent de choix face à divers stimuli, tels que les changements paysagers ou climatiques ainsi que les contraintes spatiales imposées par l'homme (zonages et autres méthodes de contention). Si l'on reprend l'exemple du comportement des loups américains face aux clôtures à drapeaux évoqué précédemment, certains individus ont pu faire le choix délibéré de les franchir à partir d'une analyse coûts/bénéfices (autrement dit en comparant le risque d'être abattu ou blessé à la possibilité de se nourrir sans grand effort). Des travaux récents sur les relations anthropozoologiques cherchent à faire état de l'agentivité de l'animal (Gouabault et Michalon (dir.), 2010; Kohler (dir.), 2012). Les méthodes sont diverses et variées: Baratay (2012) se propose d'interpréter les récits mentionnant les faits et gestes de l'animal à la lumière des découvertes éthologiques; Lescureux (2010) s'intéresse aux savoirs des populations locales quant au comportement des espèces ; Campbell (2008, 2009) et Kohler (2012) privilégient l'observation directe des animaux (individuellement ou en groupe) à la lumière de grilles de lecture appliquées normalement aux humains. Les trois méthodes se valent, bien que chacune présente des risques d'erreur $d^{\prime}$ interprétation ${ }^{7}$, et peuvent être mobilisées collectivement. Elles sont toutes applicables à des réflexions d'ordre géographique notamment lorsqu'il s'agit d'analyser la façon dont les animaux se meuvent dans l'espace et se comportent vis-à-vis des propres mouvements humains.

Le troisième point concerne l'étude des effets spatiaux et scalaire des conflits. Dans le premier domaine, cela revient, par exemple, à s'intéresser aux aménagements spécifiques produits par les conflits, comme, par exemple, la "clôture à dingos " (Canis lupus dingo) coupant l'Australie en deux afin d'éviter son incursion dans les zones d'élevage (souhait des éleveurs) et lui laisser le droit d'exister loin d'elles (souhait des associations de défense des animaux: Arnould et Simon, 2007). En ce qui concerne les jeux d'échelles, il est intéressant de replacer un conflit localisé dans des perspectives plus générales. Le conflit qui oppose les éleveurs d'ovins des alpes françaises aux loups s'inscrit dans une dynamique européenne, le

\footnotetext{
${ }^{6}$ Sus scrofa

${ }^{7}$ Les éthologues ne sont pas toujours d'accord quant à la façon d'interpréter un même phénomène comme cela a été démontré par Despret (1996). Du côté des populations locales, des interprétations erronées ou stéréotypées du comportement animal sont fréquentes, surtout pour les espèces jugées inutiles ou peu attractives (travaux personnels de terrain en Amazonie, 2011, 2012). Enfin, l'observation directe selon des grilles de lectures normalement dédiées à l'homme fait courir le risque d'une projection anthropomorphique.
} 
retour de l'espèce est favorisé par des décisions communautaires (Convention de Berne, programmes Life...) et les difficultés de l'élevage ovin sont en partie liés à la réforme de la Politique Agricole Commune.

Le quatrième point a trait à l'analyse de la temporalité des conflits. Torre (op. cit) part du principe que plus un conflit s'inscrit dans la durée, plus il est important et donc potentiellement difficile à résoudre. La lutte contre les loups sur le territoire français s'inscrit dans un temps long et marquée par des épisodes plus ou moins sanglants, encore très présents dans la mémoire collective (De Planhol, 2004 ; Moriceau, 2007). Aller à l'encontre de cette animosité séculaire pour faire en sorte que le loup trouve à nouveau sa place sur le territoire français risque donc de s'avérer difficile.

\section{La prise en compte des perceptions : les travaux sur la « juste place » des animaux}

De nombreux auteurs évoquent un écart entre les dommages réels que provoquent les animaux et la façon dont ils sont vécus par les populations humaines, soulignant l'importance d'étudier les filtres culturels, religieux ou idéels à l'origine de cette altération de la perception (Madden, op. cit ; Dickman, 2010). La manière dont les humains perçoivent leur environnement et ses différentes composantes a fait l'objet de nombreux travaux en géographie dite culturelle. Actuellement, la branche culturelle des travaux géographiques consacrés aux animaux est la plus développée avec des recherches portant sur des sujets très divers tels que: la construction sociale de la frontière entre (animaux) humains et animaux (non humains); les valeurs symboliques et identitaires liées à certaines espèces (Lassiter, 2002) ; et, enfin, la façon dont les sociétés réservent, autorisent ou refusent l'accès de certains espaces à certaines espèces. Comme nous l'avons évoqué précédemment, les conflits hommes/animaux sauvages sont éminemment liés à cette dernière composante, le conflit naissant lorsque l'animal sort ou s'apprête à sortir de l'espace réel ou symbolique qui lui a été alloué par un individu ou un groupe d'individus.

Cet espace octroyé à l'animal a été conceptualisé sous le terme de " juste place " (Mauz, 2002b). Cette " juste place " est définie en fonction de la nature de l'espèce (" une espèce montagnarde doit se trouver en montagne et non en plaine »), son comportement (" une espèce sauvage doit être craintive et par conséquent loin des hommes et de leurs établissements ") mais aussi la nature de l'espace (" une montagne sera d'autant plus authentique que l'on y observera des espèces montagnardes en pleine possession de leurs moyens). La question des représentations occupe une place importante dans la définition de cette "juste place", si bien qu'elle est souvent stéréotypée et ne correspond pas au comportement réel de l'espèce ou à l'avancée des connaissances scientifiques dans ce domaine. Mauz cite l'exemple d'ongulés sauvages (chamois, bouquetins ${ }^{8}$ ) que les populations des Alpes françaises assignent à la haute montagne car des années de chasse et de conduite des troupeaux les y ont circonscrits. Or, depuis quelques années, la réduction des activités humaines dans les étages inférieurs et les mesures de protection à leur égard les ont rendues moins craintives, d'où des sorties en dehors de ce que l'on considérait être leur « habitat naturel ». Si ces incursions en vallée ou à proximité des exploitations agricoles peuvent sembler logiques aux yeux des écologues et des naturalistes (processus de

\footnotetext{
${ }^{8}$ Respectivement Rupicapra rupicapra et Capra Ibex.
} 
conquête d'une niche écologique laissée vacante), elles sont moins bien perçues par les acteurs locaux qui y voient une "déviance " de l'animal et une multitude de problèmes (risque de collision sur la route, consommation de la végétation normalement destinée aux animaux d'élevage). Même si tous ne font pas référence à l'expression de " juste place ", de nombreux travaux géographiques se sont intéressés aux discours et représentations liés à l'animal, notamment pour analyser les jeux d'acteurs entre les défenseurs et les opposants à différentes espèces problématiques (Mounet, op._cit ; Benhammou, op. cit, Boussin, 2008, Poinsot, 2008). D'autres recherches ont insisté davantage sur les filtres formant ou déformant la perception des espèces animales en tentant d'analyser le poids des trajectoires socioprofessionnelles et éducatives, de la culture locale (religion, folklore) mais aussi des sensibilités collectives et individuelles (Naughton-Treves, 2002).

Bien que de nombreuses disciplines mènent des recherches sur la façon dont les conflits et les espèces sont perçus (anthropologie, psychologie, philosophie) et que le traitement géographique de la question soit relativement développé, de nombreux éléments restent à découvrir dans ce domaine. À titre d'exemple, au Brésil, les études sur la perception des animaux problématiques et leur " juste place " sont pour l'instant assez lacunaires et limités à quelques espèces emblématiques comme le jaguar ${ }^{9}$ (Zimmermann et al., 2005). $S^{\prime}$ intéresser aux opinions en circulation sur les espèces problématiques est fondamental afin de pouvoir cibler les actions d'information et de sensibilisation qui permettront peu à peu de désamorcer les conflits. Cela revient notamment à relativiser les attaques de carnivores sur des humains à la lumière de statistiques diverses (données environnementales ou historiques) afin d'en souligner le caractère exceptionnel et d'écorner l'image abondamment répandue de " machines à tuer » (Thirgood et al., op. cit ; Moriceau, 2007 ).

\section{L'analyse de la dimension spatiale des conflits et leur mise en carte}

Si la question des représentations liées à l'animal a bien été analysée par les géographes, paradoxalement, leur engagement dans l'analyse de la dimension spatiale des conflits et les réalisations cartographiques à cet égard demeurent limités. Ces travaux sont pourtant d'une importance capitale que ce soit pour mettre en exergue les facteurs géographiques pouvant expliquer les causes d'un conflit ou pour aider à sa résolution (orienter la mise en place d'aménagements spécifiques comme des couloirs à faune, des clôtures ou des zonages...).

L'importance et la gravité des conflits varient en fonction de différents critères ayant une dimension spatiale. Les densités humaines et animales, la distance entre les établissements humains et les zones occupées par les animaux tout comme l'existence de ressources ou de portions d'espace convoitées par les deux parties (points d'eau, forêts parcourues pour l'extractivisme) constituent des facteurs souvent mis en avant par la littérature technicoscientifique pour expliquer les causes d'un conflit. Les dynamiques paysagères et les formes d'occupation du sol ont également leur importance : certaines cultures comme le piment ou le poivre repoussent un grand nombre d'herbivores problématiques, la présence d'une clairière régulièrement nettoyée entre un fragment forestier et une zone cultivée peut dissuader les espèces forestières d'y pénétrer. D'autres facteurs doivent être pris en compte comme la qualité des habitats. Par exemple, la raréfaction des proies naturelles ainsi que la

\footnotetext{
${ }^{9}$ Panthera onca.
} 
dégradation de la végétation ou des cours d'eau sont considérés comme des facteurs explicatifs des attaques de félins sur le bétail et les humains (Löe \& Roskaff, 2004). II est également primordial de considérer les formes d'occupation de l'espace des espèces problématiques. Certaines sont fortement attachées à des lieux particuliers : les bandes de babouins ${ }^{10}$ qui s'attaquent aux plantations sylvicoles ou aux ovins en Afrique occupent les mêmes sites de repos d'une nuit à l'autre. Ainsi pour éviter les conflits, on peut choisir de les déloger ou de maintenir les activités humaines à une distance raisonnable déterminée en fonction du rayon d'éloignement moyen de ces sites de repos (Lamarque, op. cit). Les jeux de compétition entre espèces peuvent également fournir des informations utiles pour la résolution des conflits. Au Kenya, les éléphants et les babouins s'évitent soigneusement (Buard, 2005), par conséquent, il est possible de choisir l'espèce avec laquelle la cohabitation demeure la plus aisée et tenter de faciliter son maintien sur la zone afin de résoudre indirectement le conflit avec la seconde. Enfin, il est important de noter que les changements saisonniers ont également leur importance: les lions du Kenya semblent davantage s'attaquer au bétail et aux humains lors des époques de haute précipitation, le contraire est observé pour les crocodiles.

Ces différents critères ont été mis en évidence majoritairement par les sciences naturelles, surtout ceux qui ont trait aux effets des conditions d'habitat sur les stratégies animales, et par quelques géographes (Naughton-Treves, op. cit ; Campbell, 2008, 2009). L'intérêt de la géographie est de pouvoir mettre en carte les conflits afin de souligner les configurations spatiales à l'origine des tensions, mais aussi de définir des éventuelles zones de vulnérabilité aux conflits. Le travail de Master de Buard (op. cit) constitue un des rares exemples ayant cherché à croiser dans un SIG différents critères (densité humaine, accès à une source d'eau, types d'habitat privilégié par les espèces animales) afin de cartographier les risques de conflits entre humains, éléphants, porcs sauvages et babouins. Cette relative discrétion des géographes dans ce domaine est regrettable. Si la cartographie des conflits hommes/faune sauvage est jugée ardue par certains auteurs (Arnould, 2009), l'exemple donné par Buard prouve que cela n'est pas impossible. Comme les sciences naturelles montrent un intérêt certain pour la mise en évidence de la dimension spatiale de ces conflits (Smith et Kalski, 2000), il est possible que la participation de la géographie croisse dans les années à venir. Comme cette question a également trait à la manipulation de données écologiques (définition de la qualité de l'habitat ou des compétitions entre espèces) et éthologiques (comportement de l'animal dans un espace donné), cette contribution de la géographie ne pourra être réalisée sans un étroit dialogue avec ces disciplines.

\section{Conclusion}

L'objectif de cet article était de montrer que les conflits hommes/animaux sauvages connaissaient de nombreuses variations en fonction des configurations territoriales rencontrées et que, par conséquent, cet objet d'étude pouvait être de l'intérêt de la géographie. Les différentes sciences s'intéressant auxdits conflits sont à la recherche de méthodes et de grilles d'analyse afin d'en expliquer les causes profondes et proposer des solutions viables sur le plan social et écologique. Nous avons alors mis en évidence les

\footnotetext{
${ }^{10}$ Papio hamadyras
} 
différents apports de la géographie des conflits environnementaux, de la géographie culturelle et les potentialités de l'analyse spatiale dans ce domaine. Ces apports ont été présentés de façon cloisonnée pour les besoins de la démonstration, cependant, il est évident que pour une appréhension globale des conflits, une analyse conjointe des configurations territoriales, des perceptions et de leurs critères spatiaux doit être privilégiée. Cette étude globale des conflits hommes/animaux sauvage présente de nombreux défis pour la discipline géographique. Le premier est d'opérer un rapprochement entre des branches disciplinaires qui ont eu dans le passé des tendances à se séparer et qui, aujourd'hui encore, éprouvent des difficultés à dialoguer. II est par exemple primordial que la géographie culturelle et la biogéographie se rencontrent, car, comme le souligne Campbell (op. cit), les idées circulant sur les espèces ont un impact direct sur leur répartition dans l'espace et le temps. En France, des siècles d'opinions négatives à l'encontre du loup n'ont-elles pas conduit son éviction du territoire français et aux tentatives de contention actuelles ? Le second est de réussir à cartographier les conflits, autrement dit, réussir à mettre en exergue les zones de tension entre les territoires humains et ceux des animaux, ces derniers ne pouvant être appréhendés qu'avec le concours d'autres disciplines telles que l'écologie ou l'éthologie. Le troisième est de parvenir à considérer les stratégies individuelles et collectives des animaux pour en faire un acteur à part entière et non un simple élément contextuel. $\mathrm{Ce}$ qui revient par exemple à se demander comment l'animal adapte son comportement aux faits et gestes des humains et comment il peut, en retour, influer sur ces derniers. À titre d'exemple, dans un article de 2008, Campbell évoquait la capacité de certains oiseaux de petite taille à s'attirer les faveurs des humains qui les nourrissent afin de renverser l'ordre naturel des choses (infériorité face aux oiseaux de plus grande taille ou plus agressifs). Pour l'instant, le point de vue et les stratégies de l'animal sont quelque peu délaissés dans les travaux des sciences humaines portant sur les conflits hommes/faune sauvage, mais cela peut constituer un nouveau pan de recherche dans les années à venir.

\section{Bibliographie}

Arnould P. (2009) "Le loup nouveau est arrivé ", Géocarrefour, vol. 83, 3, http://geocarrefour.revues.org/6974.

Arnould P. \& Simon L. (2007) Géographie de l'environnement, Paris, Belin, 303 p.

Baratay E. (2012) Le point de vue de l'animal. Une autre version de l'histoire, Paris, Seuil, 389 p.

Benhammou F. (2007) Crier au loup pour avoir la peau de l'ours. Une géopolitique locale de l'environnement à travers la gestion et la conservation des grands prédateurs en France, Thèse de doctorat en sciences de l'environnement, Ecole Nationale du Génie Rural, des Eaux et Forêt de Paris, $665 \mathrm{p}$.

Benhammou F. \& Dangléant C. (2009) Ours, lynx, loup : une protection contre nature, Toulouse, Milan éditions, $117 \mathrm{p}$.

Bertrand C. \& G. (2002) Une géographie traversière, l'environnement à travers territoires et temporalités, Paris, Arguments, $360 \mathrm{p}$.

Binot A., Castel V. et Caron A. (2006) "L'interface faune-bétail en Afrique subsaharienne ", Sécheresse, vol._17, 1\&2, pp. 349-361.

Boussin L. (2008) « L'animal sauvage à travers ses représentations, l'exemple du chevreuil en Limousin ", Annales de Géographie, n 663, pp. 48-61. 
Buard E. (2005) Spatial analysis of human-wildlife conflicts in transmara district, Kenya, Mémoire de Master, Cranfield University at Silsoe, $66 \mathrm{p}$.

Campbell M. (2008) "An animal geography of avian feedings habits in Petersborough, Ontario», Area, vol. 40, 4, pp. 472-480.

Campbell M. (2009) «Repositioning zoogeography within the nature-culture borderlands: An animal geography of reptiles in southern Ghana», Applied geography, vol. 29, 2, -pp. 260-268, Collomb G. (2009) « Sous les tortues, la plage ? Protection de la nature et production des territoires en Guyane ", Ethnologie française, vol. 1, 39, pp. 11-21.

De Planhol X. (2004) Le paysage animal, l'homme et la grande faune, une zoogéographie historique, Paris, Fayard, $1127 \mathrm{p}$.

Despret V. (1996) Naissance d'une théorie écologique: la danse du cratérope écaillé, Le Plessis-Robinson, Synthélabo, $231 \mathrm{p}$.

Distefano E. (2005) Human-Wildlife Conflict worldwide: collection of case studies, analysis of management strategies and good practices,

http://www.fao.org/SARD/COMMON/ecg/1357/en/HWC final.pdf.

Dickman A. J. (2010) «Complexity of conflict: the importance of considering social factors for effectively resolving human wildlife conflict», Animal conservation, vol.13, pp. 458-466.

Emel J. \& Urbanik J. (2010) «Animal Geographies: Exploring the Spaces and Places of Human-Animal Encounters", in DeMello, Teaching the Animal: Human-Animal Studies across the disciplines, New York, Lantern Books, pp. 9-23.

Inskip Ch. \& Zimmermann A. (2009) «Human-felid conflict: a review of patterns and priorities worldwide», -Oryx, vol. 43, 1, pp. 18-34.

Gouabault E. \& Michalon J. (dir.) (2010) «Relations anthropozoologiques ", Sociétés, n¹08, $138 \mathrm{p}$.

Goeury D. (2010) «Le Ladakh, royaume du développement durable ? ", Revue de géographie alpine, vol. 98, 1, http://rga.revues.org/1100.

Guyot S. (2003) « Les conflits environnementaux à St Lucia (Afrique du Sud), entre protection et exclusion ", Annales de Géographie, n 634, pp. 608-627

GTA (2008) O fim da floresta ? A devastação das unidades de conservação e terras indígenas no estado de Rondônia, Rapport du Grupo de Trabalho Amazônico du Rondônia, 62 p.

Johansson T. (2009) "The Spatial Dimension of Human-Wildlife Conflicts - Discoveries of New Animal Geography " in Donert K., Ari Y., Attard M., O'Reilly G. et Schmeinck D., Geographical Diversity. Proceedings of the HERODOT Conference in Ayvalik, pp.257-265.

Kohler F. (dir.) (2012) « Sociabilités animales », Études rurales, n¹89, 219_p.

Lamarque F. (2010) "Les conflits humains-faune en Afrique. Causes, conséquences et stratégies de gestion ", Études FAO Forêts, $n^{\circ} 157,125$ p.

Larson C. L. (2008) Separating people and wildlife. Zoning as conservation strategy for large carnivores, Honors Theses, 245, http://digitalcommons.colby.edu/honorstheses/245.

Laslaz L. (2005) Les zones centrales des Parcs nationaux alpins français (Vanoise, Écrins, Mercantour), des conflits au consensus social ? Contribution critique à l'analyse des processus territoriaux d'admission des espaces protégés et des rapports entre sociétés et politiques d'aménagement en milieux montagnards, Thèse de doctorat en géographie, Université de Savoie,- $644 \mathrm{p}$.

Lassiter U. (2002) " Le contexte spatial de l'authenticité des animaux ", Espaces et Sociétés, $\mathrm{n}^{\circ} 110-111$, pp. 41-57.

Lescureux N. (2010) « Le bon, la brute et le fantôme ", Annales de la Fondation Fyssen, vol. 24, pp. 1-18. 
Löe J. \& Röskaft E. (2004) "Large carnivores and human safety a review», AMBIO: A Journal of the Human Environment, 33, 6 L pp. 283-288.

Luxereau A. (2004) "Des animaux ni sauvages ni domestiques, les "girafes des Blancs" au Niger ", Anthropozoologica, 39, 1, pp. 289-300.

Madden -F. (2004) « Creating coexistence between humans and wildlife global perspectives and local efforts to adress human wildlife conflicts ", Human Dimensions of Wildlife, $n^{\circ}$, 247--257.-

Mauz I. (2002a) Gens, cornes et crocs. Relations hommes-animaux et conceptions du monde, en Vanoise, au moment de l'arrivée des loups, Thèse de doctorat en sciences de l'environnement, CEMAGREF, Grenoble, $513 \mathrm{p}$.

Mauz I. (2002b) "Les conceptions de la juste place des animaux dans les Alpes françaises ", Espaces et sociétés, $\mathrm{n}^{\circ} 110-111$, pp $129-145$.

Milhaud O. (2005) "Y a-t-il une géographie du territoire animal ? ", Compte rendu du café géographique du 22 mars 2005, http://www.cafe-geo.net/article.php3?id article=612

Mounet C. (2007) Les territoires de l'imprévisible. Conflits, controverses et "vivre ensemble" autour de la gestion de la faune sauvage. Le cas du loup et du sanglier dans les Alpes françaises, Thèse de doctorat en géographie, université Grenoble 1, $564 \mathrm{p}$.

Moriceau J.-M. (2007) Histoire du méchant loup, 3000 attaques sur I'homme en France XV' $X X^{e}$ siècle, Paris, Fayard, $622 \mathrm{p}$.

Naughton-Treves L. (2002) «Wild Animals in the Garden: Conserving Wildlife in Amazonian Ecosystems ", Annals of the Association of American Geographers, vol. 92, 3, pp. 488-506.

Philo Ch. \& Wilbret Ch. (2000) Animal spaces, beastly places: new geographies of humananimal relations, London, Routledge, $311 \mathrm{p}$.

Poinsot Y. (2008) « Les enjeux géographiques d'une gestion durable de la faune sauvage en France ", Annales de Géographie, $n^{\circ} 663$, pp. 26-47.

Proust J. (2000) « L'animal intentionnel », Terrain, $n^{\circ} 34$, pp. 23-36.

Shivik J. A., Treves A. et Calahan P. (2003) « Nonlethal techniques for managing predation: Primary and secondary repellents ", Conservation Biology, 17, 6, pp. 1531-1537

Smith R. J. \& Kasiki S. M (2000) A spatial analysis of human-elephant conflict in the tsavo ecosystem, Kenya, Rapport au groupe de spécialistes des éléphants africains de l'UICN, 82 p.

Sogbohossou E.A et al. (2011) «-Human-carnivore conflict around Pendjari biosphere reserve, northern Benin", Oryx, vol. 45, 4, pp. 569-578.

Staszak J. F. (dir.) (2002) « La place de l'animal », Espaces et Sociétés, n 110/111.

Thirgood S., Woodroffe R. et Rabinowitz A. (2005) « The impact of human-wildlife conflicts on human lives and livelihoods " in Woodroffe et al., People and wildlife, conflict or coexistence ?, Cambridge, Cambridge University Press, pp. 13-26.

Torre A. (2010) "Conflits environnementaux et territoires" in Zuindeau B. (dir.), Développement Durable et territoire, Presses Universitaires du Septentrion, 518 p.

Woodroffe R., Thrirgood S. et Rabinowitz A. (2005) People and wildlife, conflict or coexistence ?, Cambridge, Cambridge University Press, $497 \mathrm{p}$. 